\title{
La biopsia oral en el contexto del precáncer y del cáncer oral
}

\author{
Seoane $\mathrm{JM}^{*}$, González-Mosquera A*, Velo-Noya J*
}

\section{RESUMEN}

Es este artículo se revisa el papel de la biopsia oral en el diagnóstico de lesiones precancerosas y en el diagnóstico precoz del cáncer oral. Se discuten diferentes técnicas, procedimientos, materiales, indicaciones y aspectos quirúrgicos. Se propone efectuar biopsias incisionales en lesiones malignas y sospechosas de malignidad, en tanto se preconizan biopsias escisionales, cuando el tamaño lo permita, en lesiones precancerosas..

Palabras clave: Biopsia oral, Precáncer oral, Cáncer oral.

\section{SUMMARY}

This article reviews the paper of oral biopsy on the precancerous lesions diagnosis and on the oral cancer early diagnosis. Different techniques, procedures, materials, indications and other surgical aspects are debated. It proposes to do incisional biopsies on malignant lesions and on malignant suspicious lesions, while doing excisional biopsies on precancerous lesions when the size allows it.

Key words: Oral biopsy, Oral precancer;, Oral cancer.

Fecha de recepción: Diciembre 2007.

Aceptado para publicación: Diciembre 2008.

* Unidad de Cirugía Oral y Maxilofacial. Facultad de Medicina y Odontología de Santiago de Compostela. España.

Seoane JM, González-Mosquera A, Velo-Noya J. La biopsia oral en el contexto del precáncer y del cáncer oral. Av. Odontoestomatol 2008; 24 (1): 89-96.

\section{INTRODUCCIÓN}

Si nos preguntamos, trabajando en este escenario, ¿cuál es el papel de la biopsia?, sin duda se trata de establecer el diagnóstico definitivo de estas lesiones, evitando así el retraso asignado al profesional en el diagnóstico de esta patología.

Existen lesiones precancerosas o de cáncer oral en las que mediante el estudio de su aspecto e historia clínica es posible plantear un diagnóstico clínico de presunción, sin embargo el estudio histopatológico es usualmente imprescindible para establecer un diagnóstico definitivo. En esta línea, la biopsia oral es un procedimiento quirúrgico encaminado a obtener tejido de un organismo vivo, para su estudio microscópico, básicamente con una finalidad diagnóstica (1).

Otros objetivos de este procedimiento son (2):

- Establecer un diagnóstico definitivo de la lesión en base a su aspecto histológico. 
- Establecer un pronóstico para las lesiones malignas y premalignas.

- En algunos casos, facilitar la prescripción de tratamientos específicos.

- Contribuir en la evaluación de la eficacia de los tratamientos.

- Constituir, en cualquier caso, un documento con evidente valor médico-legal.

\section{INDICACIONES}

La biopsia oral está indicada para la confirmación de lesiones sospechosas de malignidad, lesiones precancerosas como las leucoplasias o eritroplasias y las ulceraciones orales sin causa evidente. En este sentido, se encuentran indicadas en lesiones aparentemente inflamatorias que no mejoran o desaparecen en dos semanas, tras eliminar los irritantes locales. También deberían biopsiarse las lesiones que interfieren con la función oral, lesiones de etiología poco clara, particularmente cuando se asocian a dolor, parestesia o anestesia y lesiones intersticiales en la musculatura lingual, bucal y labial $(1,2,3,4)$.

\section{¿Cuando no es necesaria la biopsia oral?}

No deben biopsiarse estructuras normales, lesiones leves del desarrollo (Estomatitis geográfica, Tori ... etc), lesiones claramente irritativas y/o traumáticas que responden a la remoción de irritantes locales. No deben hacerse biopsias incisionales en lesiones angiomatosas, ni tampoco en lesiones infecciosas (gingivitis, abscesos periodontales, pericoronaritis) que pueden responder a tratamientos específicos $(2,5)$.

\section{TIPOS DE BIOPSIA $(1,2)$ :}

De acuerdo a los procedimientos aplicados, las biopsias orales pueden clasificarse según:

\section{Las características de la lesión}

- Biopsias directas: Cuando la lesión se encuentra posicionada sobre la mucosa oral y es fácilmente accesible. Este es el caso de los precánceres orales y de las lesiones primarias neoplásicas.
- Biopsias indirectas: cuando la lesión se encuentra recubierta de una mucosa aparentemente normal.

\section{El área de la remoción quirúrgica}

- Biopsia incisional consiste en la remoción de una muestra representativa de la lesión y tejido adyacente normal con el objetivo de establecer un diagnóstico definitivo de forma previa al tratamiento.

- Biopsia escisional se trata de la remoción completa de la lesión con un doble objetivo diagnóstico y terapéutico. Tan solo es posible cuando el tamaño lesional permite la escisión completa con márgenes adecuados sanos.

\section{Según el momento de la biopsia respecto a la intervención quirúrgica}

- Preoperatoria.

- Intraoperatoria.

- Postoperatoria con la finalidad de evaluar la eficacia del tratamiento.

La biopsia oral en lesiones sospechosas de malignidad ha generado una fuerte controversia sobre los conocimientos y la capacidad legal de los dentistas para efectuarla, la idoneidad de referir o biopsiar, y sobre la elección de los diferentes materiales y técnicas para minimizar los artefactos en las muestras $(2,5,6)$.

\section{¿PUEDEN LOS ODONTÓLOGOS Y ESTOMATÓLOGOS HACER BIOPSIAS DE LESIONES ORALES SOSPECHOSAS DE MALIGNIDAD?}

Ante esta pregunta existe un triple posicionamiento. De una parte, se considera que la biopsia bajo anestesia local efectuada por dentistas constituye el procedimiento diagnóstico más importante en el diagnóstico precoz del cáncer oral (2). Con idéntico argumento, la Academia Americana de Patología Oral considera que la biopsia se encuentra "en el foco" de la práctica odontológica (6). Sin embargo, otros autores preconizan para los dentistas ante una lesión sospechosa de precáncer o cáncer oral una 
actitud no intervencionista: "No pánico, no biopsiar y referir inmediatamente”... (4).

En cualquier caso, la legislación española y comunitaria otorga capacidad legal, tanto a odontólogos como a estomatólogos, para llevar a cabo este tipo de práctica. Además, en países del primer mundo los retrasos diagnósticos constituyen el 30\% de las reclamaciones por mala práctica y los errores diagnósticos encabezan los motivos de litigios contra dentistas (5).

¿Saben hacer biopsias orales los odontólogos y estomatólogos en España?

En España existen pocos estudios que permitan conocer la proporción de odontólogos/estomatólogos que efectúan biopsias orales de forma rutinaria . Los datos existentes sugieren que esta proporción oscila según áreas geográficas y colectivos explorados entre un 24 y un 50\%, incrementándose esta proporción en la última década y con los años de experiencia profesional (7-9) (Tabla 1). Además no parece existir una diferencia significativa en cuanto a la correlación clínico-patológica con otros grupos profesionales, como los cirujanos maxilofaciales, especialmente en cuanto al precáncer oral (8).

\section{¿BIOPSIAR O REFERIR?}

Aunque la técnica de biopsia oral se encuentra incluida entre las competencias profesionales del dentista general, algunos dentistas pueden sentirse más cómodos utilizando criterios adecuados de referencia de pacientes que eviten retrasos diagnósticos en este tipo de patologías.

En virtud de la baja prevalencia y las reducidas tasas de detección del cáncer oral en programas de "screening" en España, parece más adecuado promocionar programas de "screening oportunista" durante las visitas de rutina en las clínicas dentales o los chequeos generales de salud. (10-14). En este sentido, el screening oportunista en las clínicas dentales por parte de dentistas generalistas sobre pacientes de alto riesgo podría resultar coste-efectivo. En este sentido, el éxito de la detección precoz del cáncer oral depende de establecer con prontitud un diagnóstico de sospecha de malignidad y referir al paciente a un centro especializado para establecer un diagnóstico definitivo y el tratamiento correspondiente. (15-17). En relación con la sensibilidad y especificidad en el diagnóstico visual del cáncer oral durante el screening, los resultados publicados en la literatura presentan datos heterogéneos. De forma

\section{TABLA1.- ACTITUDES Y CONOCIMIENTOS DE LOS DENTISTAS EN ESPAÑA RESPECTO AL PAPEL DE LA BIOPSIA EN EL PRECÁNCER Y CÁNCER ORAL (8)}

\begin{tabular}{|l|c|c|c|c|c|c|}
\hline Conocimientos y actitudes & $\begin{array}{c}\text { Encuesta } \\
\mathbf{1 9 9 7}\end{array}$ & $\begin{array}{c}\text { Encuesta } \\
\mathbf{2 0 0 4}\end{array}$ & $\begin{array}{c}\text { Diferencia } \\
\mathbf{( \% )}\end{array}$ & $\begin{array}{c}\text { Chi } \\
\text { cuadrado }\end{array}$ & $\mathbf{p}$ & $\begin{array}{r}\text { IC (95\%) de } \\
\text { la diferencia }\end{array}$ \\
\hline $\begin{array}{l}\text { Presenciaron al menos } \\
\text { un cáncer oral }\end{array}$ & $114(60)$ & $32(87,5)$ & 27,5 & 8,61 & 0,00 & $12,9-42,1$ \\
\hline $\begin{array}{l}\text { Considera que la biopsia es } \\
\text { un procedimiento diagnóstico } \\
\text { rutinario }\end{array}$ & $114(66,7)$ & $32(84,4)$ & 17,7 & 3,77 & 0,05 & $2,4-32,9$ \\
\hline $\begin{array}{l}\text { Efectúa biopsias orales para } \\
\text { descartar cáncer }\end{array}$ & $114(24,6)$ & $32(50)$ & 25,4 & 7,68 & 0,00 & $6,3-44,4$ \\
\hline $\begin{array}{l}\text { Exploración sistemática } \\
\text { de tejidos blandos }\end{array}$ & $114(84,1)$ & $32(87,5)$ & 3,4 & 0,03 & 0,85 & $-9,9-16,7$ \\
\hline $\begin{array}{l}\text { Advierte sobre el alcohol } \\
\text { y el tabaco }\end{array}$ & $114(69,9)$ & $32(84.4)$ & 14,5 & 2,57 & 0,10 & $-0,6-29,0$ \\
\hline
\end{tabular}


genérica se considera que sensibilidades por encima del $80 \%$ son aceptables para el screening sistemático del cáncer oral . Sin embargo los valores de concordancia diagnóstica a menudo ocultan valores bajos de sensibilidad (capacidad del examinador de hacer una detección positiva correcta), que oscilan entre 0,60 y 0,95 (17).

La especificidad se refiere a la competencia del examinador de efectuar una detección negativa; esto es, la proporción de individuos sin patología precancerosas o de cáncer oral que han sido correctamente clasificados por los odontólogos/estomatólogos. En este sentido, es importante conocer, en la práctica general, que casos deberían ser remitidos (todas las lesiones sospechosas de malignidad y lesiones "borderline"). Una baja especificidad implicaría que pacientes con carcinomas orales no serían referidos adecuadamente para establecer el diagnóstico definitivo y el tratamiento adecuado. Estudios previos, durante los screening para el cáncer y precáncer oral han reportado una especificidad que oscila del 0,75 al 0,99 (17).

En cualquier caso, el examen visual puede ofrecer una alta capacidad de discriminación en la detección de esta enfermedad, y permite a los dentistas generalistas referir lesiones sospechosas de malignidad con alta sensibilidad y especificidad. Sin embargo, ocasionalmente se han detectado retrasos diagnósticos en pacientes referidos con lesiones sospechosas de malignidad condicionados fundamentalmente por malos protocolos de referencia, cartas de remisión a los especialistas, errores en la priorización de las citas, aspectos burocráticos etc. Por ello, es muy importante establecer protocolos estandarizados de referencia para este tipo de pacientes con la finalidad de asegurar un rápido acceso al sistema sanitario y el establecimiento de forma precoz del diagnóstico definitivo (18).

\section{¿CUÁL ES EL MATERIAL IDÓNEO EN LAS BIOPSIAS ORALES? ¿CÓMO MINIMIZAR LOS ARTEFACTOS?}

Con la finalidad de obtener especímenes de calidad, libres de artefactos y que permitan al patólogo establecer un adecuado diagnóstico histológico, de forma clásica se ha utilizado el bisturí frío como material usual en la ejecución de biopsias orales. Más recientemente, el bisturí circular (punch) (Fig.1) se ha preconizado para las biopsias incisionales, especialmente las múltiples, por su sencillez en el empleo y por minimizar los artefactos en las muestras (19). Sin embargo, esta circunstancia no ha podido ser demostrada de forma concluyente en estudios experimentales (19). La proporción de artefactos en las muestras (hendiduras, hemorragias, aplastamientos y pseudoquistes) ha resultado ser similar con ambos materiales. Los puntos de sutura para fijar los especímenes y su manipulación deterioran los tejidos, especialmente los puntos superficiales (Fig.2) (19).

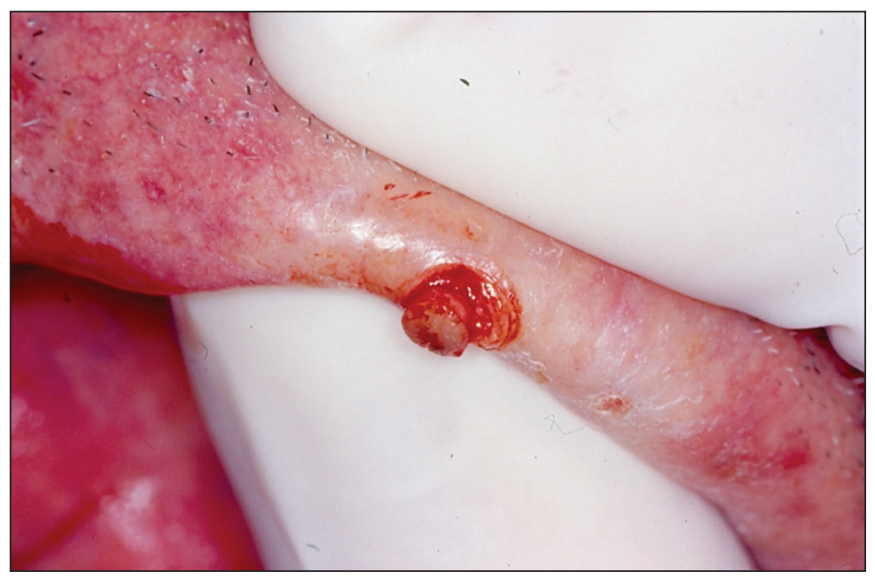

Fig. 1. Biopsia mediante punch en queilitis actínica.

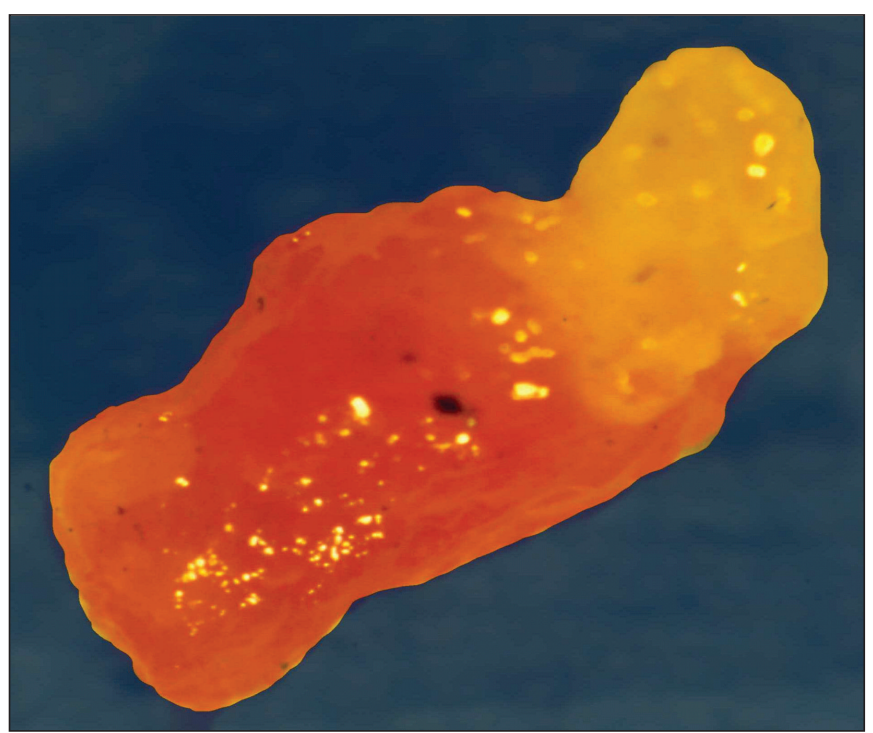

Fig. 2. Cilindro mucoso obtenido mediante punch artefactado con las pinzas de biopsia. 
De forma específica debe evitarse el empleo de LASER para la toma de biopsias orales sobre lesiones precancerosas o de cáncer oral, dado que se han descrito cambios displásicos, atipias citológicas, hipercromatismo- pleomorfismo y elongación nuclear asociados al empleo de LASER ( $\mathrm{CO}_{2}$. Nd:YAG), esta circunstancia induciría una fuerte confusión al patólogo $(20,21)$. Además existen distintos materiales auxiliares a la biopsia oral, fundamentalmente se han descrito pinzas fenestradas -Pinzas B del Prof. Bermejo (22), pinzas de chalazión (23) (Fig.3) de empleo en áreas perilabiales y lengua móvil, y el punch rotatorio para facilitar la biopsia en áreas de difícil acceso (24).

Si nos preguntásemos, ¿̇cuáles son los errores más frecuentes que deben ser evitados en las biopsias orales? Los puntos clave a considerar serían el evitar:

- Presionar intempestivamente la muestra con las pinzas, especialmente si son dentadas y artefactar la muestra.

- Infiltrar la solución anestésica dentro de la lesión.

- Aplicar o pincelar productos en la superficie de la lesión que pueda generar modificaciones titulares.

- Usar un volumen insuficiente de fijador.

- Incluir en la muestra material proveniente de restauraciones dentales, cálculo o polvo de los guantes quirúrgicos.

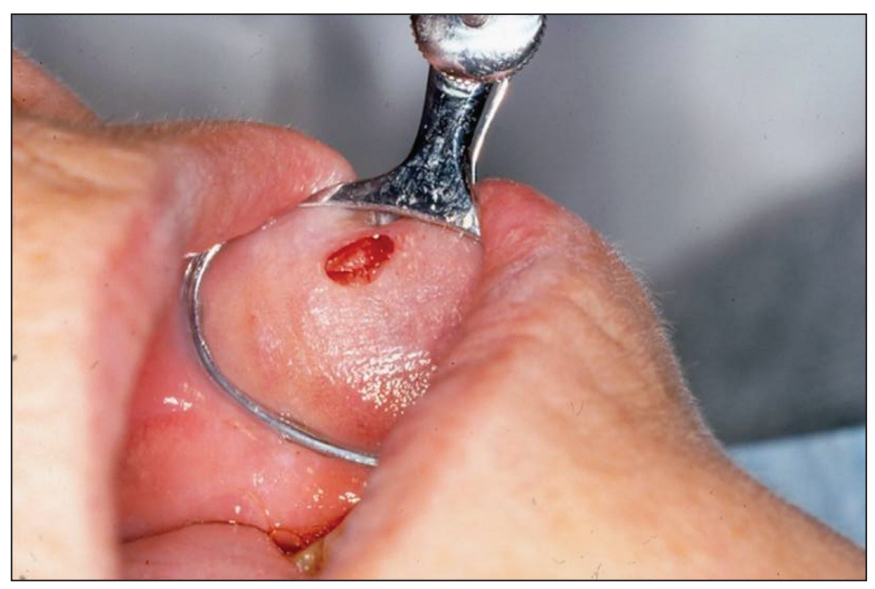

Fig. 3. Pinzas de chalazion como material auxiliar en la biopsia oral.
- Tomar material no representativo o una cantidad insuficiente en extensión y en profundidad.

\section{¿BIOPSIAS INCISIONALES VERSUS ESCISIONALES EN EL DIAGNÓSTICO PRECOZ DEL CÁNCER ORAL?}

A pesar de que debido a la heterogeneidad tumoral, es bien conocido el efecto de infradiagnóstico e infravaloración de la severidad de las lesiones displásicas y de los carcinomas orales precoces cuando efectuamos biopsias incisionales y de la existencia de diseminación sanguínea de células procedentes del carcinoma oral $(25,26)$, el incremento del crecimiento local de la neoplasia primaria $(27,28)$ y de la mayor incidencia de metástasis cervicales en estadios I y II, en pacientes previamente diagnosticados mediante biopsias incisionales. De forma genérica, se admite que todas las lesiones sospechosas de malignidad requieren una biopsia para establecer un diagnóstico definitivo. En estas circunstancias, las biopsias incisionales procurando mostrar el espesor tumoral, son las más adecuadas (Fig.4). Esta actitud se encuentra reforzada por el hecho de que biopsias escisionales sobre carcinomas orales efectuadas por dentistas generalistas sin criterio oncológico, van a dejar restos microscópicos tumorales y destrucción de los márgenes del carcinoma (29). Además, Pacientes con cáncer oral manejados mediante biopsias escisionales inadecuadas suelen precisar una reescisión y un abordaje cervical en dependencia del estadio y de la profundidad de invasión.

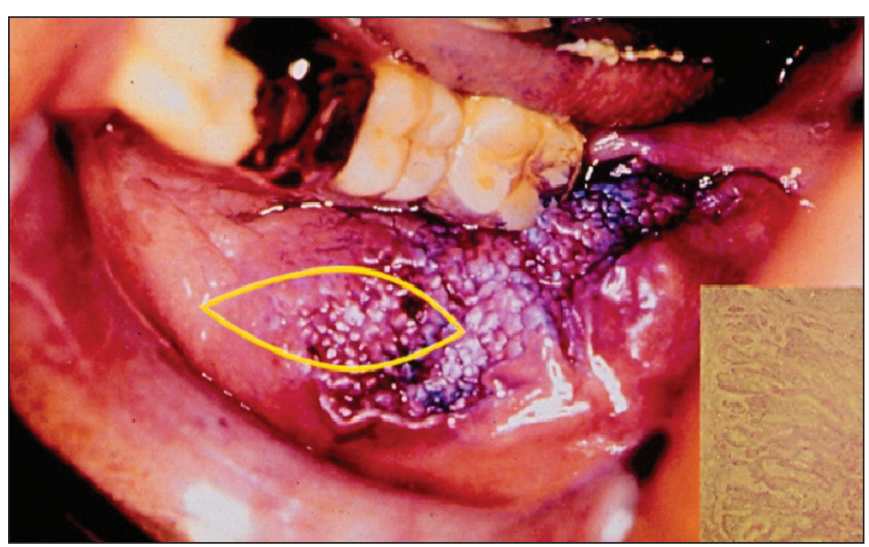

Fig. 4. Biopsia incisional para el diagnóstico de un carcinoma de mejilla. 


\section{TÉCNICA QUIRÚRGICA. PRINCIPIOS GENERALES DE LA BIOPSIA (2)}

De forma previa al procedimiento quirúrgico debe efectuarse una historia clínica que recoja aspectos sobre la lesión referentes al tamaño, forma, color, textura, consistencia, tiempo de evolución, síntomas asociados y presencia de adenopatías locorregionales, con la finalidad de establecer un diagnóstico clínico y un diagnóstico diferencial. Además, el paciente debería recibir información al respecto evitando generar ansiedad para obtener el consentimiento informado adecuado a esta técnica.

\section{Aspectos generales de la técnica quirúrgica}

- Son preferibles técnicas de bloqueo troncular sobre las técnicas infiltrativas.

- Son preferibles incisiones paralelas a los nervios y los vasos, generalmente se utilizan incisiones elípticas que facilitan la sutura.

- En lesiones precancerosas de pequeño tamaño $(<1 \mathrm{~cm})$, estarían indicadas biopsias escisionales. Lesiones mayores propiciarían biopsias incisionales que incluyan tejido lesional representativo, tejido transicional y márgenes sanos.

- En las lesiones malignas o con alta sospecha clínica de malignidad es obligatorio efectuar biopsias incisionales.

- Los especímenes deben ser orientados mediante un hilo de sutura e introducidos en un frasco con una solución fijadora adecuada (formol al 10\%).

El número y la localización de las biopsias debe decidirse en base a la apariencia clínica de la lesión. En lesiones heterogéneas con diferentes aspectos clínicos deben tomarse varias muestras; generalmente el empleo de colorantes vitales como Azul de Tolouidina resulta útil como procedimiento auxiliar para elegir las áreas más relevantes a biopsiar (30).

La muestra obtenida con tejido patológico y sano debe de presentar suficiente tamaño, de manera que se eviten problemas. Hay que considerar que procesos como la fijación del espécimen puede reducir sensiblemente su tamaño. Además, las muestras pequeñas son difíciles de orientar y de manipular.

\section{BIOPSIA ORAL MEDIANTE BISTURÍ FRÍO $(1,2)$}

Con la finalidad de conseguir una adecuada visibilidad necesitamos una buena iluminación y separadores del tipo Farabeuf o retractores de labios y mejillas, además de aspiración quirúrgica. El material fundamental para abordar esta técnica consiste en: Material de anestesia local, bisturíes (no. 15, 11), pinzas de mosquito, pinzas de Allis, sutura atraumática de 2/0 a 5/0, gasas estériles y frasco con solución fijadora. Está técnica quirúrgica puede simplificarse en 6 etapas: selección del área a biopsiar, preparación del campo quirúrgico, anestesia local, incisión, manipulación del espécimen y sutura de la herida quirúrgica.

\section{SELECCIÓN DEL ÁREA A BIOPSIAR}

Son preferibles biopsias escisionales en lesiones precancerosas de pequeño tamaño $(<1 \mathrm{~cm})$, por el contrario, en caso de lesiones con alta sospecha de malignidad o lesiones precancerosas de gran tamaño son preferibles biopsias incisionales. La tinción con azul de toluidina puede resultar útil en la elección de las áreas lesionales más representativas. Dado que es capaz de identificar zonas con alto riesgo de sufrir cambios displásicos, identificar lesiones con alto riesgo de malignización o carcinomas asintomáticos.

\section{PREPARACIÓN DEL CAMPO QUIRÚRGICO}

Ocasionalmente puede emplearse compuestos con amonio cuaternario. Aunque de forma usual se utilizan enjuagues con clorhexidina al 0,12-0,20\%. No deben utilizarse antisépticos que contengan Yodo.

- Anestesia local: Deben evitarse anestesias infiltrativas intralesionales que introducen artefactos importantes en los especímenes.

- La Incisión: Se inmoviliza el tejido con una pinzas de Allis sin dientes y se efectúa una incisión elíptica "en gajo de naranja" que incluya tejido representativo y tejido sano. Si existen diversas lesiones deben efectuarse varias biopsias.

- Manipulación de los tejidos: Las muestras deben tratarse cuidadosamente evitando artefactos 
(hendiduras, pseudoquistes, aplastamientos, hemorragias y fragmentación). Algunos autores han propuesto colocar los especímenes en papel estéril mirando hacia arriba para evitar la distorsión y el "curling" en los márgenes de las muestras pequeñas. Como agente fijador se utiliza formalina al $10 \%$, dado que induce pocas alteraciones titulares y ocasionalmente el etanol al $70 \%$ podría ser usado. Sin embargo, deben evitarse el alcohol metílico o isopropílico, el suero salino y el agua destilada, en virtud de las alteraciones inducidas por estos agentes. El volumen de las soluciones fijadoras debe exceder 10 a 20 veces el volumen de la muestra. En cualquier caso el material remitido al patólogo debe acompañarse de una breve historia clínica, con el diagnóstico de presunción y otros diagnósticos diferenciales; así como un diagrama explicativo del área biopsiada.

- Sutura: Debería lograr una hemostasia adecuada, facilitar la curación de la herida y en caso de ser no reabsorbible debe eliminarse después de 6 a 8 días.

Se concluye esta revisión resaltando la necesidad de reforzar aspectos educacionales para fomentar el empleo rutinario de las biopsias orales como procedimiento diagnóstico entre los odontólogos y estomatólogos. La posibilidad de emplear diferentes materiales (bisturí frío o punch), junto con materiales auxiliares (pinzas fenestradas y material rotatorio), evitando materiales que produzcan daño térmico (electrobisturí y LASER) para proporcionar especímenes de alta calidad al patólogo. Se propone efectuar biopsias incisionales en lesiones malignas y sospechosas de malignidad, en tanto se preconizan para las lesiones precancerosas, las biopsias escisionales, cuando el tamaño y las circunstancias clínicas lo permitan.

\section{BIBLIOGRAFÍA}

1. Mota-Ramirez A, Silvestre FJ, Simó JM. Oral biopsy in dental practice Med Oral Patol Oral Cir Bucal 2007;12(7):E504-10.

2. OM Handbook de la European Association of Oral Medicine disponible en: http://www. eaom.net/app/prvt/VediNotizia.d/Notizia-92.
3. Kahn MA, Lynch DP, Turner JE, Mincer HH. The dos and don'ts of an oral mucosal biopsy performed by the general dentist. J. Tenn. Dent. Assoc 1998;78:28-31.

4. McAndrew PG. Oral cancer biopsy in general practice. Br Dent J 1998;185:428.

5. Rapp C. Oral Cancer malpractice claims increasing. Today's FDA 2005;17:37-8.

6. Melrose RJ, Handlers JP, Kerpel S et al. The use of biopsy in dental practice: The position of American Academy of Oral and Maxillofacial Pathology. Gen Dent 2007; September/October 457-61.

7. Epstein JB, Gorsky M, Fischer D, Gupta A, Epstein M, Elad S. A survey of the current approaches to diagnosis and management of oral premalignant lesions. J Am Dent Assoc 2007; 138:1555-62.

8. Seoane J, Warnakulasuriya S, Varela-Centelles P, Esparza G, Dios PD. Oral cancer: experiences and diagnostic abilities elicited by dentists in north-western Spain. Oral Dis 2006;12:487-92.

9. Lopez-jornet P, Velandrino Nicolas A, MartinezBeneyto Y, Fernandez-Soria M. Attitude towards oral biopsy among general dentist in Murcia. Med Oral Patol OralCir Bucal 2007;12:E116.

10. Jullien JA, Downer MC, Speight PM, Zakrzewska JM. Evaluation of health care worker's accuracy in recognising oral cancer and pre-cancer. Int Dent J 1996; 46:334-9.

11. Warnakulasuriya KAAS, Johnson NW. Dentists and oral cancer prevention in the UK: opinions, attitudes and practices to screening for mucosal lesions and to counseling patients on tobacco and alcohol use: baseline data from 1991. Oral Dis 1999;5:10-4. 26.

12. Smart CR. Screening for cancer of the aerodigestive tract. Cancer 1993;72:1061-5

13. Cruz GD, Ostroff JS, Kumar JV, Gajendra S. Preventing and detecting oral cancer: oral health 
care providers' readiness to provide health behavior counseling and oral cancer examinations. J Am Dent Assoc 2005;136:594-682.

14. Field EA, Morrison T, Darling AE, et al. Oral mucosal screening as an integral part of routine dental care. Br Dent J 1995;179:262-6.

15. Leao JC, Góes P, Sobrinho CB, Porter S. Knowledge and clinical expertise regarding oral cancer among Brazilian dentists. Int $J$ Oral Maxillofac Surg 2005;34:436-9.

16. Ikeda N, Isii T, Lida S, Kamiya Y, et al. A study of mass screening for oral mucosal diseases of adults. Japan. J Oral Maxillofac Surg 1998;34:2394-402.

17. Moles DR, Downer MC, Speight PM. Meta-analysis of measures of performance reported in oral cancer and precancer screening studies. Br Dent J 2002;192:340-4.

18. Singh P, Warnakulasuriya S. The two-week wait cancer initiative on oral cancer; the predictive value of urgent referrals to an oral medicine unit. Br Dent J 2006;201:717-2

19. Seoane J, Varela-Centelles P, Ramirez JR, Romero MA, De La Cruz A. Artefacts produced by suture traction during incisional biopsy of oral lesions. Clin Otolaryngol allied Sci 2002; 27: 549-53.

20. Eversole LR . Laser artefacts and diagnostic biopsy. Oral Surg Oral Med Oral Pathol 1997; 83:639-41.

21. Gould AR. Early detection of oral premalignant disease and oral cancer: Refining the process. Oral Surg Oral Med Oral Pathol 2002;94:397-8.

22. Bermejo-Fenoll A, Lopez-jornet P, Jimenez-Torres MJ, Camacho-Alonso F, Orduña-Domingo A. Biopsy of the buccal mucosa in oral lichen planus: the traditional method versus the use of newpressure forceps. J Am Dent Assoc 2007;138:957-6.

23. Seoane J, Varela-Centelles PI, Diz-dios P, Romero $M$. Use of chalazion forceps to ease biopsy of minor salivary glands. Laryngoscope 2000;110:486-7.
24. Seoane J, Varela-Centelles P, Diz-Dios P, Limeres J. Powered rotary scalpel for biopsies in difficult areas of the oral cavity. J Oral Maxillofac Surg 2006;64:563-4.

25. Pentenero M, Carrozzo M, Pagano M, Galliano D, Broccoletti R, Scully C, Gandolfo S. Oral mucosal dysplastic lesions and early squamous cell carcinomas: underdiagnosis from incisional biopsy. Oral Dis. 2003 Mar;9(2):68-72. 29. Convissar RA. Laser biopsy artifact. Oral Surg Oral Med Oral Pathol Oral Radiol Endod. 1997;Nov;84(5):458.

26. Kusukawa J, Suefuji Y, Ryu F, Noguchi R, Iwamoto O, Kameyama T. Dissemination of cancer cells into circulation occurs by incisional biopsy of oral squamous cell carcinoma. J Oral Pathol Med. 2000;Aug;29(7):303-7.

27. Safour IM, Wood NK, Tsiklakis K, Doemling DB, Joseph G. Incisional biopsy and seeding in hamster cheek pouch carcinoma. J Dent Res. 1984;Sep;63(9):1116-20.

28. Tsiklakis K, Wood NK, Toto PD, Doemling DB.Hamster cheek pouch carcinoma: effect of incision and cortisone on growth, invasion, and metastasis. J Dent Res. 1986;Jun;65(6):927-31.

29. Bailey JS, Blanchaert RH Jr, Ord RA.Management of oral squamous cell carcinoma treated with inadequate excisional biopsy. J Oral Maxillofac Surg. 2001;Sep;59(9):1007-10; discussion 1011.

30. Epstein JB, Sciubba J, Silverman SJr, Sroussi HY. Utility of toluidine blue in oral premalignant lesions and squamous cell carcinoma: continuing research and implications for clinical practice. Head and Neck 2007;20:948-58.

\section{CORRESPONDENCIA}

Juan M. Seoane Lestón

Unidad de Cirugía Oral y Maxilofacial.

Facultad de Odontología.

Entrerrios, s/n

Santiago de Compostela.Coruña. España

Mail: jseoanel@usc.es 